\title{
Preface: metals in the brain
}

\author{
Astrid Sigel · Helmut Sigel
}

Received: 27 November 2010/Accepted: 10 December 2010/Published online: 22 January 2011

(c) Springer-Verlag 2011

At the beginning of the last century a newborn in Europe had a life expectancy of about 45 years. Now, approximately 100 years later, this number has increased in the West to more than 80 years [1]. The reasons for this extended life span are manyfold, the more important ones being improved hygiene, progress in medicine, a reduced mortality of mother and child, control of epidemics, and better food provisions [2]. As we all know, this has led to a tremendous growth of the world population: About 1 billion $\left(=10^{9}\right)$ people inhabited the Earth in 1804 ; it took nearly 125 years to double this number, by 1974 it was 4 billion, and the estimate for 2025 is 8 billion [1].

However, this story of success has a seamy side: because of the increased life span, cancer and other age-related diseases, especially those connected with neurodegeneration, mainly manifested in the brain, are getting more and more prominent. To this category belong, among others, Alzheimer's disease (AD), Parkinson's disease (PD), amyotrophic lateral sclerosis (ALS), Huntington's disease (HD), transmissible spongiform encephalopathy (prion disease; Creutzfeldt-Jakob disease), neurodegeneration with brain iron accumulation (NBIA; formerly Hallervorden-Spatz disease), Friedreich's ataxia, progressive supranuclear palsy (PSP), and multisystem atrophy (MSA) [3].

The best known of these debilitating illnesses are certainly Alzheimer's [3] and Parkinson's [3, 4] diseases. About 29 million people worldwide suffered from Alzheimer's disease in 2009; this is about $60 \%$ of those being affected by dementia. Computer models predict that the

\footnotetext{
A. Sigel · H. Sigel $(\bowtie)$

Department of Chemistry, Inorganic Chemistry, University of Basel, Spitalstrasse 51, 4056 Basel, Switzerland e-mail: Helmut.Sigel@unibas.ch
}

number of patients with AD will increase to 106 million by 2050 [1], which means one per 85 persons-even if this number should be off by a factor of 2, one out of 170 persons is still truly frightening!

It is an old wisdom that metals are indispensable for life; in fact, several of them, like sodium, potassium, and calcium, are easily discovered in living matter [5]. However, the role of metals and their impact on life remained largely hidden until inorganic chemistry and coordination chemistry experienced a pronounced revival in the middle of the last century. The experimental and theoretical tools created and their application to biochemical problems led to the development of the field or discipline Bioinorganic Chemistry, which was established by 1970. It also became known as Inorganic Biochemistry and more recently as Biological Inorganic Chemistry [3].

At present there is substantial evidence that metal ions play a significant role in the pathogenesis and pathophysiology of neurodegenerative diseases, which reveal themselves mainly in the brain. This is not surprising because the brain utilizes metal ions for a myriad of biochemical reactions. The metal-related disorders are caused (1) by defects in metal ion transport or homeostasis, (2) by toxicological exposure to metals, and (3) they are associated with metalloprotein aggregation and/or misfolding and oxidative reactions [3]. This organizational frame is certainly helpful, yet to assign a given illness to a distinct category is not easy because of our lack in understanding the mechanisms involved in a given disease, though misfolded proteins are often implicated. Of course, toxic metal ions like mercury or cadmium are easily categorized, but with essential metal ions like copper, iron, zinc, or manganese, which may be toxic as well, matters are considerably more difficult and we are only at the brink of understanding the fine line between essentiality and toxicity. There is an urgent need to unravel the 
mechanisms involved in these neurodegenerative diseases; only once these are understood, is there hope for the development of effective drugs.

Jawaharlal Nehru, the first Prime Minister of free India, having in mind the difficulties India was facing, made a statement in 1961 [6] that does in a shortened form also hold for the difficulties in treating neurodegenerative disorders: "It is Science alone that can solve the problems... Who indeed could afford to ignore Science today? At every turn we have to seek its aid... The future belongs to Science and those who make friendship with Science." Therefore, the editors of this special issue of Chemical Monthly, devoted to metals in the brain must be congratulated for composing this issue and it is hoped that their and the authors' efforts have an impact on pertinent research, not only by established but also by younger scientists because new ideas are needed. It is evident that the mentioned diseases, which are increasingly affecting mankind, are closely linked to the role metal ions are playing in the brain. Clearly, huge efforts by devoted scientists will be needed to overcome the obstacles, and this also means that politicians need to provide the necessary financial support without a top-down policy, otherwise the disaster is programmed. It is our sincere hope that this issue is a strong stimulus for future research!

\section{References}

1. Wikipedia (2010) http://www.wikipedia.org. Accessed 26 Oct 2010

2. Perutz MF (1982) Ging's ohne Forschung besser? Der Einfluss der Naturwissenschaften auf die Gesellschaft. Wissenschaftliche Verlagsanstalt, Stuttgart, pp 1-54

3. Sigel A, Sigel H, Sigel RKO (eds) (2006) Metal ions in life sciences, neurodegenerative diseases and metal ions, vol 1. Wiley, Chichester, pp 1-463

4. Grayson M, Schnabel J et al (2010) Outlook: Parkinson's disease. Nature 466(7310):S1-S19 (on p S1 under "Collection" five more pertinent articles from 2009 and 2010 are cited)

5. Sigel H, Sigel A (2000) J Indian Chem Soc 77:501

6. Ram Atma (1961) Proc Natl Inst Sci India 27A:531 (see also [2] on page 5) 